\title{
Measuring system of the NEVOD-EAS array
}

\author{
S.S. Khokhlov ${ }^{*}$, , M.B. Amelchakov ${ }^{a}$, N.V. Ampilogov ${ }^{a}$, I.I. Astapov ${ }^{a}$, \\ N.S. Barbashina ${ }^{a}$, A.G. Bogdanov ${ }^{a}$, A. Chiavassa ${ }^{b, c}$, N.N. Kamlev ${ }^{a}$, V.V. Kindin ${ }^{a}$, \\ R.P. Kokoulin ${ }^{a}$, K.G. Kompaniets ${ }^{a}$, V.Yu. Kutovoy ${ }^{a}$, O.I. Likiy ${ }^{a}$, G. Mannocchi ${ }^{d}$, \\ V.V. Ovchinnikov ${ }^{a}$, A.A. Petrukhin ${ }^{a}$, O. Saavedra ${ }^{b}$, G. Trinchero ${ }^{d}$, V.V. Shestakov ${ }^{a}$, \\ I.A. Shulzhenko ${ }^{a}$, V.V. Shutenko ${ }^{a}$, I.I. Yashin ${ }^{a}$ \\ ${ }^{a}$ National Research Nuclear University MEPhI (Moscow Engineering Physics Institute), \\ Moscow 115409, Russian Federation \\ ${ }^{b}$ Dipartimento di Fisica dell' Università di Torino, 10125 Torino, Italy \\ ${ }^{c}$ INFN - Sezione di Torino, 10125 Torino, Italy \\ ${ }^{d}$ Istituto di Fisica dello Spazio Interplanetario - INAF, 10133 Torino, Italy \\ E-mail: SSKhokhlov@mephi.ru
}

NEVOD-EAS array which is now under construction in the National Research Nuclear University MEPhI (Moscow, Russia) is designed for the independent estimation of characteristics of the extensive air showers registered by the Unique Scientific Facility NEVOD in the "knee" region of the primary cosmic ray energy spectrum. The work describes the features of the NEVOD-EAS measuring system which ensures the shower registration, data acquisition, cluster synchronization and events selection according to the data of the separate clusters of the setup. The results of the scintillation counter elements testing and characteristics of the first cluster of the array are discussed.

The 34th International Cosmic Ray Conference

30 July - 6 August, 2015

The Hague, The Netherlands

\footnotetext{
"Speaker
} 


\section{Introduction}

In the studies of muon bundles in a wide range of zenith angles and multiplicities carried out at the experimental complex NEVOD [1-3] during 2002-2010 it was shown that the intensity of muon bundles in the primary particle energy range of $10^{15}-10^{18} \mathrm{eV}$ decreases slower than its expected value and exceeds the calculations at energies above $10^{18} \mathrm{eV}$ even under the assumption of a pure iron PCR composition for all contemporary models of hadron interactions. However, the technique of primary energy estimation based on the analysis of the local density spectra of muon bundles [4] registered under different zenith angles has rather low accuracy $\left(\sigma\left(\lg E_{0}\right) \sim 0.4\right)$. This is due to the contribution of EAS of different energies registered at different (random) distances from the axis to the events with a fixed local muon density. The creation around the NEVOD experimental complex of a classic shower array for the detection of EAS in the energy range of $10^{15}-10^{17} \mathrm{eV}$ will enable to determine the size and the axis position of the showers registered in the experiment, and hence, to significantly narrow the PCR energy range in which the changes in the muon component behavior may occur.

\section{NEVOD-EAS array}

Measuring system of the NEVOD-EAS shower array [5] has a cluster organization. Each of 12 clusters consists of four detector stations formed of four scintillation counters of particles of the EAS electron-photon component and is served by a Local Post of primary data acquisition. The characteristic distance between the cluster stations is $\sim 20 \mathrm{~m}$. Distances between the clusters are $\sim 50 \mathrm{~m}$. Total area of the array is $\sim 2 \times 10^{4} \mathrm{~m}^{2}$.

\subsection{Scintillation counter and detector station}

Scintillation counter of the NEVOD-EAS array (Figure 1a) consists of the NE102A plastic scintillator with the dimensions of $800 \times 800 \times 40 \mathrm{~mm}^{3}$ and the Philips XP3462 PMT enclosed in a light-tight pyramidal housing made from stainless steel. These counters were previously used in the EAS-TOP [6] and KASCADE-Grande experiments [7]. The construction of the scintillation counter housing enables to install an additional Philips XP3462 PMT. The inner surface of the housing is painted with a diffuse-reflective coating to improve the collection of light from the flashes in the scintillator caused by the passage of particles through its sensitive volume.
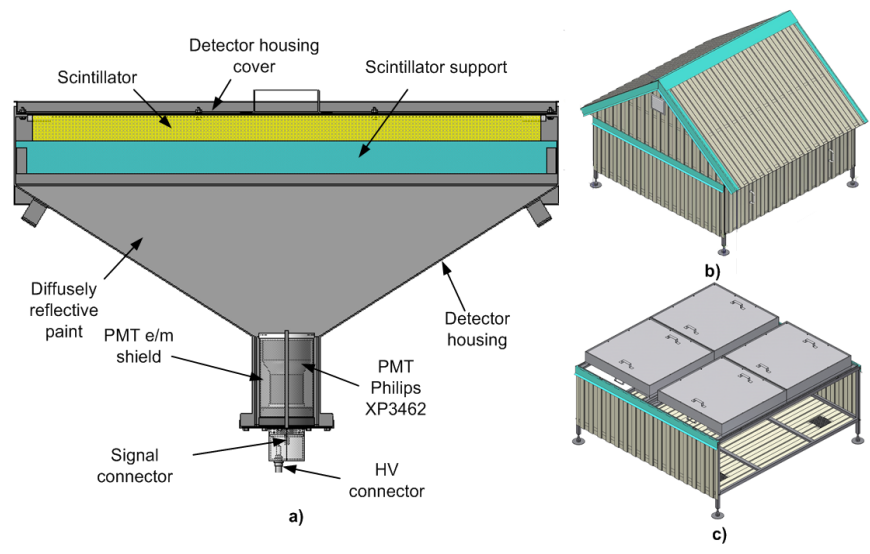

Figure 1: Counter and detector station: a) scheme of the counter; b) DS housing; c) layout of counters inside the DS housing. 
Detector station (DS) consists of 4 scintillation counters. Three counters are equipped with only a "standard" photomultiplier Philips XP3462 and the fourth one - with two PMTs (a "standard" and an "additional" ones). Four "standard" PMTs are selected and configured so that the most probable values of charge distributions of the responses of all four counters were nearly the same. These photomultipliers are used to measure the particle density and arrival time of the detected EAS. The additional photomultiplier has a dynode system gain of approximately 90 times smaller than the standard PMT. This PMT provides a wide linearity range of the measured signals at high particle densities and allows to achieve the DS dynamic range of up to 10000 particles $/ \mathrm{m}^{2}$. Counters of the DS are enclosed in special housings (Figure $1 \mathrm{~b}, \mathrm{c}$ ). Photomultipliers are powered by the DC/DC converter of low voltage $+12 \mathrm{~V}$ into high voltage in the range from -100 to $-2000 \mathrm{~V}$.

As the scintillator light yield depends on the ambient temperature, during the counter amplitude information processing it is necessary to consider the temperature coefficient. During the setup operation monitoring, temperature inside the DS is measured by means of a thermocouple.

\subsection{Cluster and Local Post of data acquisition}

Four DS placed at the vertices of a rectangle with the characteristic side lengths $\sim 20 \mathrm{~m}$ are combined into a cluster by the Local Post (LP) of data acquisition and primary processing. The main functions of the LP are receiving and digitizing of analog signals from the counters, selection of events according to intracluster triggering conditions, as well as the transfer of the amplitude and temperature information to the Central DAQ Post. Local Post (Figure 2) includes: summator-multiplexor, PET-7019 input-output module for the control of the summatormultiplexor and reception of information from the DS thermocouples, block of primary data processing, mediaconverter and power supply $( \pm 5 \mathrm{~V}, \pm 12 \mathrm{~V})$ which ensures operation of all LP elements. The LP electronics is enclosed in the water-proof thermally stabilized box.

The block of primary data processing digitizes analog signals from the detector stations of the cluster, selects events according to various intracluster triggering conditions and transmits data from cluster to the Central DAQ Post. This block includes 2 boards of amplitude analysis (BAA) and a controller based on the FPGA Xilinx Spartan-6. Controller and BAA are made according to VME standard and are mounted inside an Euromechanics $6 \mathrm{U}$ crate. Each BAA has 2 channels with 2 inputs: active and passive. The signals on each input are digitized by 12-bit FADC with a sampling frequency of $200 \mathrm{MHz}$. Registration threshold is set on the active input using 8-bit DAC. When the signal on the active input exceeds the registration threshold, data buffers of the ADCs on both inputs of the channel are stored. Control command receiving and data transmission are performed by the controller via the fiber-optic communication line. During the data transfer, each event is timestamped with an accuracy of up to $10 \mathrm{~ns}$.

Summator-multiplexor (SM) summarizes analog signals from 4 standard PMTs of the DS and has 4 channels on 4 inputs. Each channel of the SM serves one DS in which each standard PMT is connected to the corresponding input of the channel. The summed signal from 4 standard PMTs of the DS is fed to the active input of the corresponding channel of BAA. The signal from the additional PMT is fed to the corresponding passive input of the BAA. The SM 
allows to turn on a defined input of each channel for registration of amplitude and charge spectra from the selected detector of the DS during the setup operation monitoring.

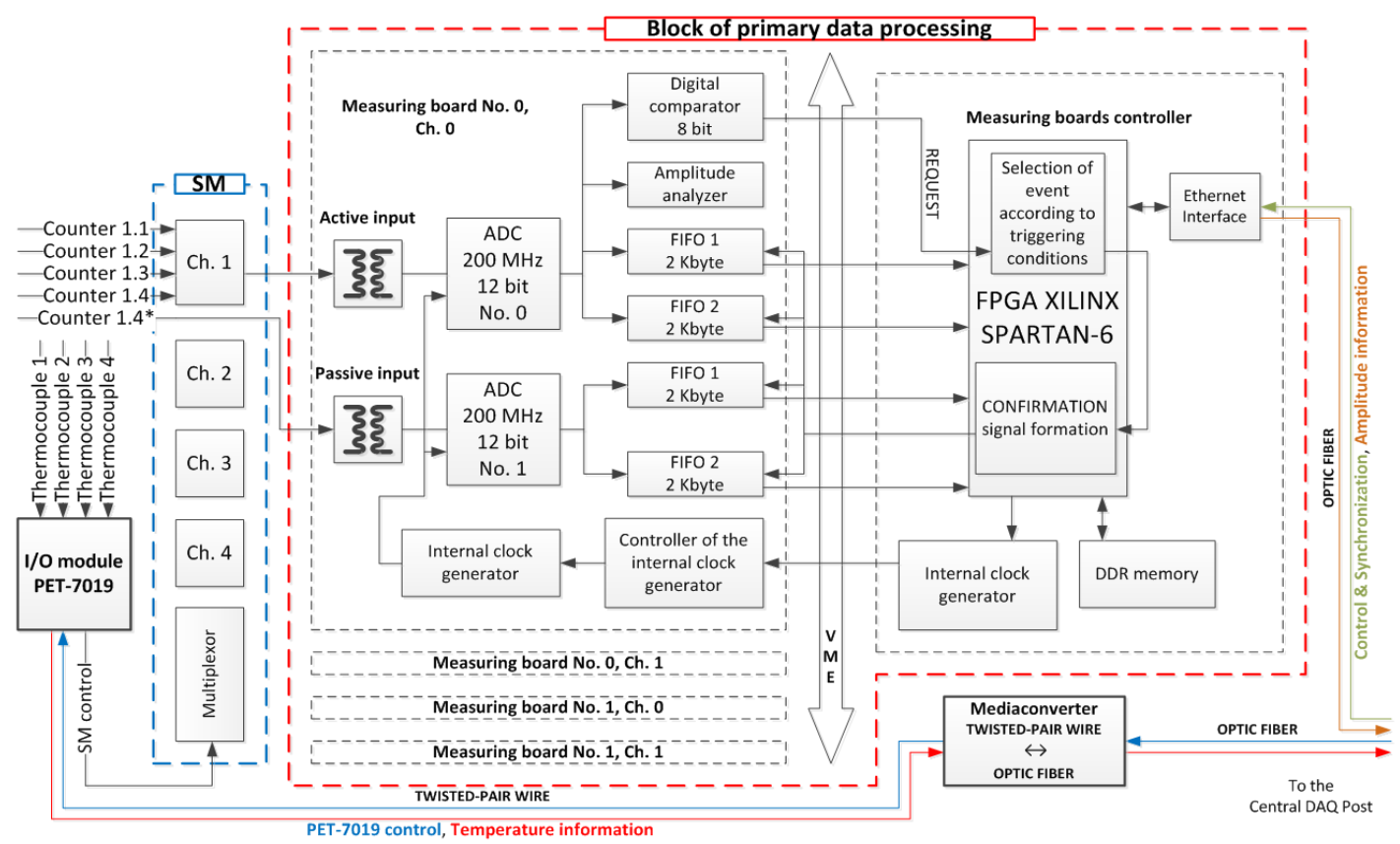

Figure 2: Electronics of the cluster Local Post.

Control of the SM is performed using the PET-7019 input-output module which has 4 discrete outputs. Also this module has 8 analog inputs for the reception of data from the DS thermocouples. Data transmission from the controller and control commands reception are performed via Ethernet network.

\subsection{Central DAQ Post}

The Central Post (CP) of the data acquisition ensures the synchronization and control of all Local Posts of the setup, as well as the receiving and storing of experimental and monitoring data. The Central Post (Figure 3) includes: personal computer, GPS/GLONASS receiver, communication unit and module of LP control and synchronization.

Communication unit of the $\mathrm{CP}$ connects the central computer with all clusters of the setup. It includes the network hub and several mediaconverters for the transmission of control commands and receiving data from the Local Posts and PET-7019 modules.

The module of LP control and synchronization consists of several special blocks - "Hosts". Each "Host" has 4 optical channels for the control and recieving of experimental data from the connected blocks of primary data processing and serves up to 4 clusters of the setup. "Host" has a 10/100TX port for the connection to CP communication unit.

Information about registered events is processed by the computer with a special software and is recorded in files that store event timestamps and parameters of signals from the detector stations of triggered clusters.

Time synchronization of clusters is performed by using the synchronization module and global positioning system (GPS/GLONASS). GPS receiver connected to the synchronization module generates the PPS signal with a frequency of $1 \mathrm{~Hz}$. Upon receiving of the PPS signal, the synchronization module sends the timestamps with the duration of $10 \mathrm{~ns}$ (one clock cycle) to 
the "Hosts" connected to it. These timestamps are used to synchronize the internal clock generator of each "Host". Then each "Host" synchronizes the connected Local Posts via fiberoptic communication line. Thus, the synchronous operation of all electronic components of the setup on a single clock frequency of $100 \mathrm{MHz}$ is achieved. The synchronization accuracy of NEVOD-EAS clusters is $10 \mathrm{~ns}$.

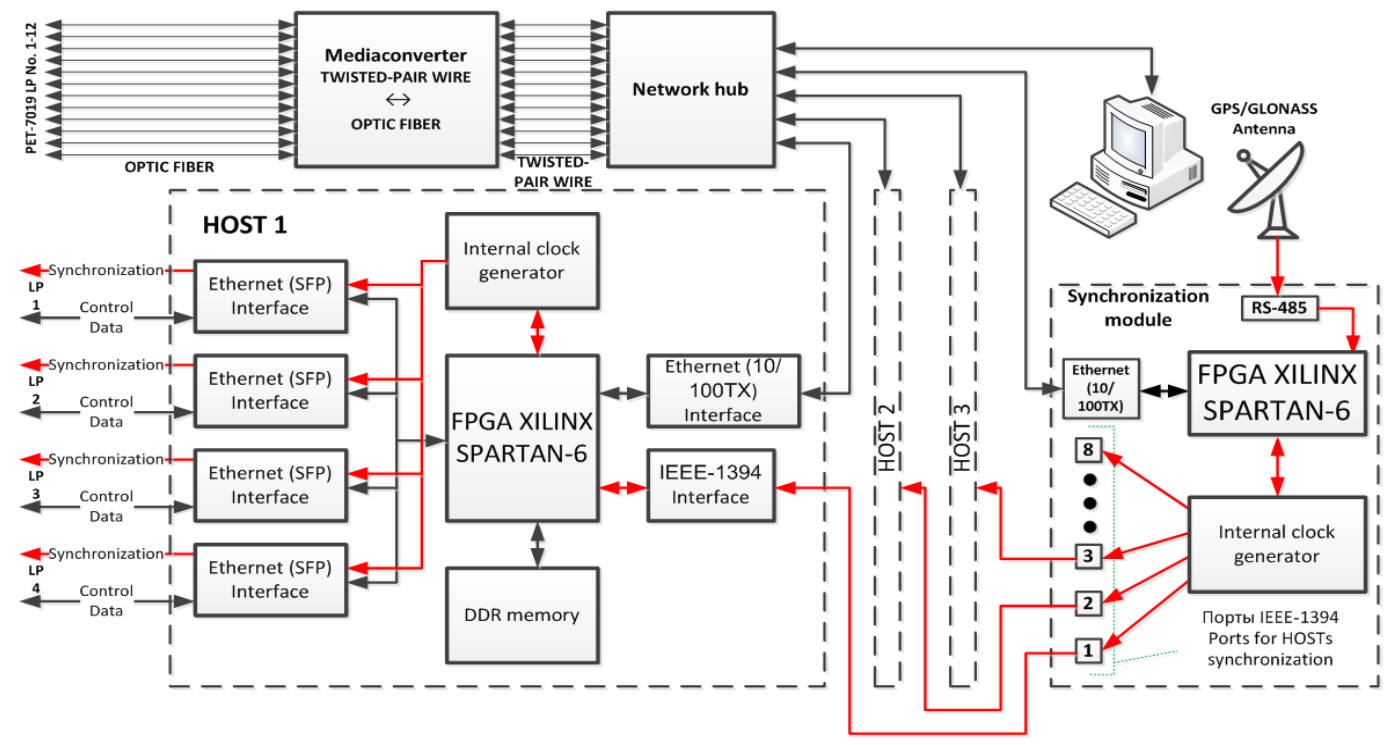

Figure 3: Electronics of the Central DAQ Post.

\section{Scintillation counter characteristics}

At the preliminary stage of the NEVOD-EAS creation, the characteristics of photomultiplier tubes and scintillators have been studied, as well as the counters have been assembled and adjusted.

\subsection{Characteristics of scintillators and PMTs}

During the Philips XP3462 PMT testing the following characteristics have been studied: the high voltage providing a gain of the photomultiplier dynode system $\sim 2 \times 10^{6}$; the spread of the PMT triggering delays (jitter) during its operation in the single photoelectron mode; the linearity range; the relative sensitivity (the response of the PMT to the photocathode illumination with a fixed intensity); the dependence of the dark noise counting rate on the registration threshold.
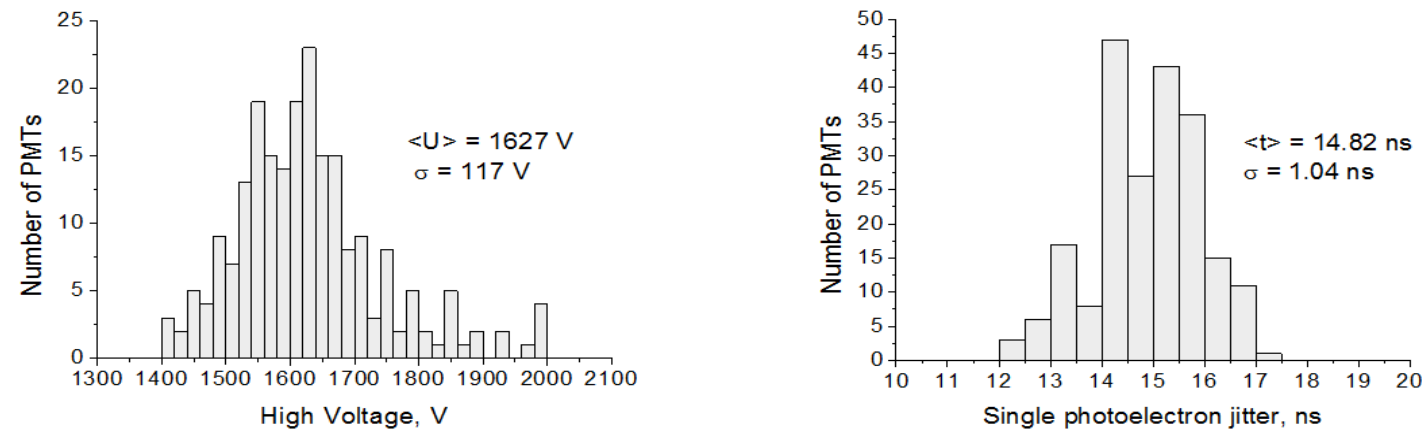

Figure 4: Distributions of the PMTs high voltage providing the PMT gain $\sim 2 \times 10^{6}$ (left) and of the PMTs single photoelectron time jitter (right). 
At the initial stage of each PMT testing, the value of high voltage providing the gain of the PMT $\sim 2 \times 10^{6}$ was determined using the technique of single photoelectron illumination of the PMT photocathode. The other characteristics were investigated at the high voltage defined at the initial stage. Figures 4, 5 and 6 (left) show distributions of 240 tested PMTs in the high voltage, single photoelectron jitter, upper limit of linearity range, relative (to the complete sample average) sensitivity of the photocathode and dark noise counting rate at the fixed threshold $(10 \mathrm{mV})$.
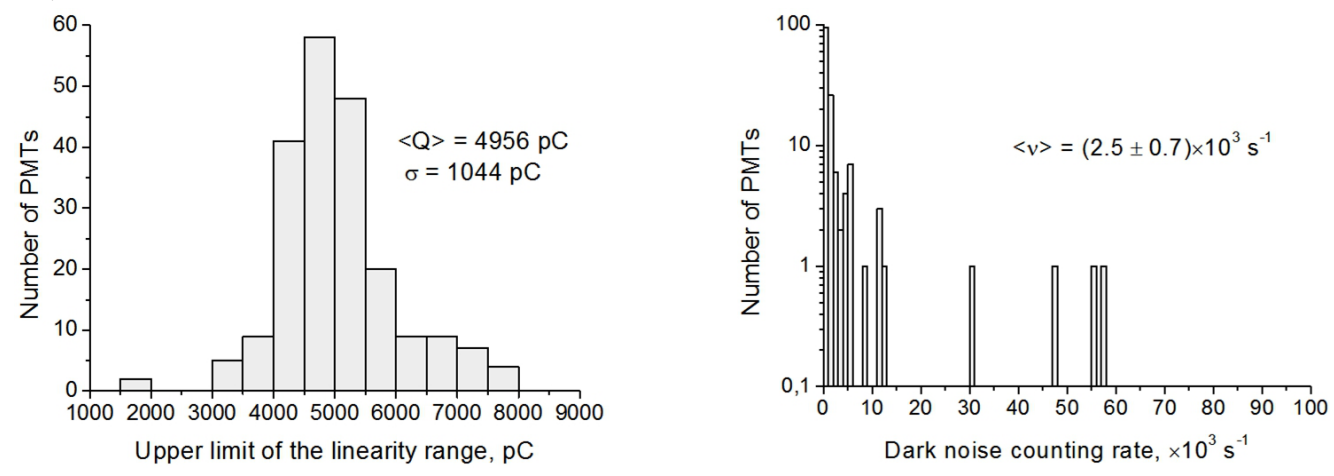

Figure 5: Distributions of the PMTs upper limit of linearity range (left) and of the PMTs dark noise counting rate (right).
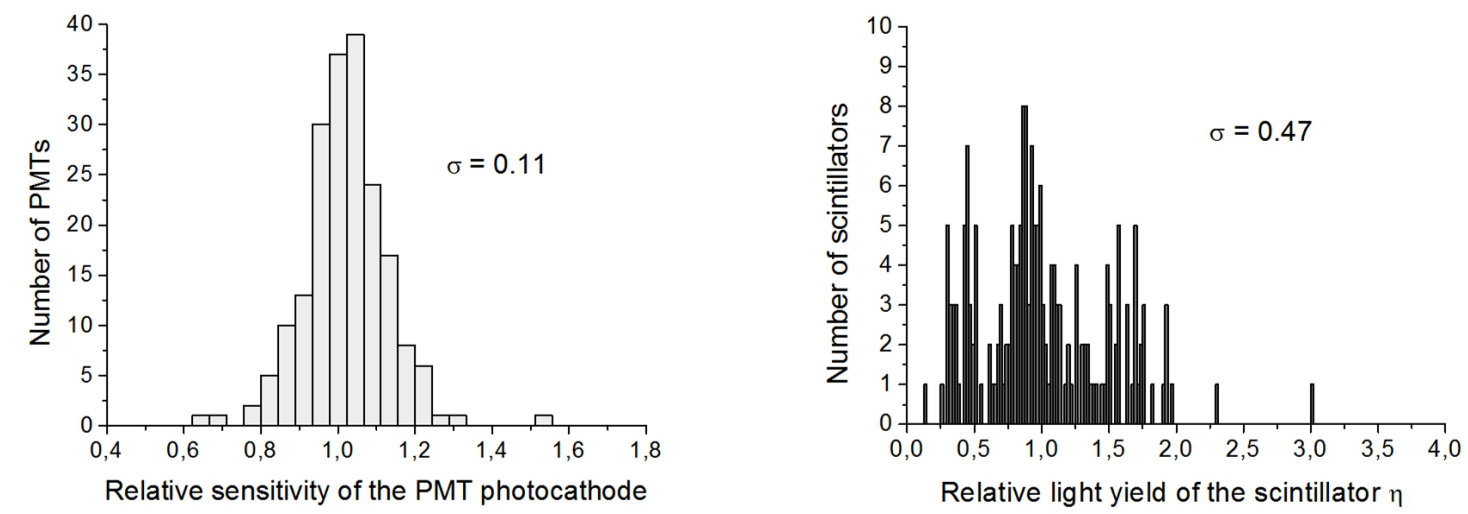

Figure 6: Distributions of the PMTs relative photocathode sensitivity (left) and of the relative light yield of the scintillators (right).

During the scintillator plate testing, the charge spectrum of counter response to the passage of near-vertical muons selected by the muon telescope has been measured. The muon telescope consists of two scintillation counters with dimensions $10 \times 10 \mathrm{~cm}^{2}$ placed in the central area of the studied scintillator. Figure 6 (right) shows the distribution of the most probable values of the measured charge spectra for 192 scintillators divided by the complete sample average charge of the muon peak (distribution of the relative light yield of the scintillators).

\subsection{Assembling and adjustment of the scintillation counters}

The assembling of the NEVOD-EAS is performed using the following principle: a lower light yield of the scintillator (relative to the complete sample average) should be compensated by a higher sensitivity of the PMT (relative to the complete sample average), and a lower PMT sensitivity should be compensated by a higher scintillator light yield. The selection of scintillators and photomultipliers is based on the distributions shown in figure 6 . 


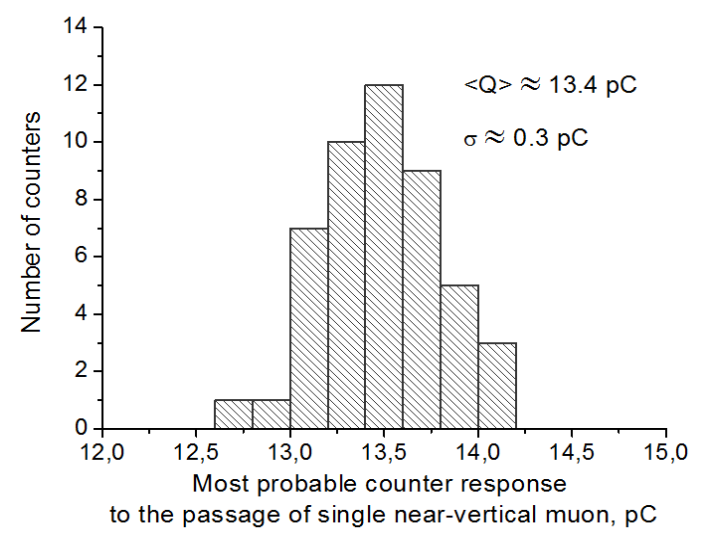

Figure 7: Distribution of the most probable responses of 48 adjusted counters to the passage of single muon.
After the assembling, the most probable response of the counter to the passage of a single muon is adjusted to the value of $\sim 13 \pm 1$ $\mathrm{pC}$ by varying the PMT supply voltage. Such response value provides the dynamic range of the counter of up to $\sim 100$ detected particles (vertical equivalent muons).

Currently, 48 NEVOD-EAS scintillation counters are assembled and adjusted. Figure 7 shows the distribution of the most probable responses of adjusted counters to the passage of single muons. The average value is $\sim 13.4 \mathrm{pC}$. The total spread does not exceed $\sim 1.4 \mathrm{pC}$.

\section{Characteristics of the NEVOD-EAS cluster}

After the adjustment of the first 16 scintillation counters, a test cluster of the NEVODEAS array was assembled and launched. Temporal characteristics of the cluster were studied in 2 configurations. In the experimental hall of the NEVOD complex the detector stations were placed in the following way: primarily, stations were installed at the vertices of a rectangle with dimensions of $10.6 \mathrm{~m} \times 12.5 \mathrm{~m}$, and then station 2 was moved close to the station 1 for estimating of the accuracy of the station response time determination.The signals from the DS counters were fed to the SM inputs, and then to the active inputs of the BAA. The 4-fold coincidence of the DS within a time gate of $50 \mathrm{~ns}$ which is determined by the distance between the stations located on the rectangle diagonal $(\sim 16.4 \mathrm{~m})$ was used as an internal trigger.

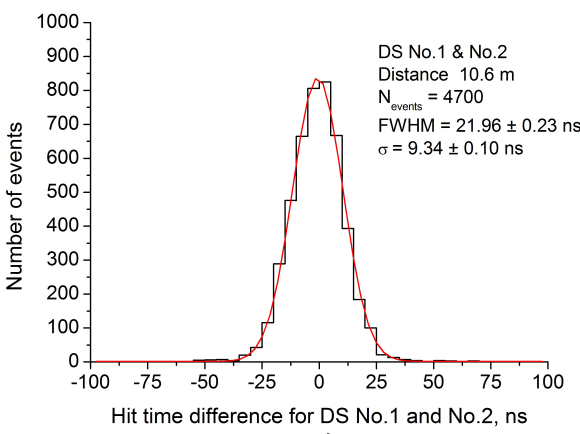

a)

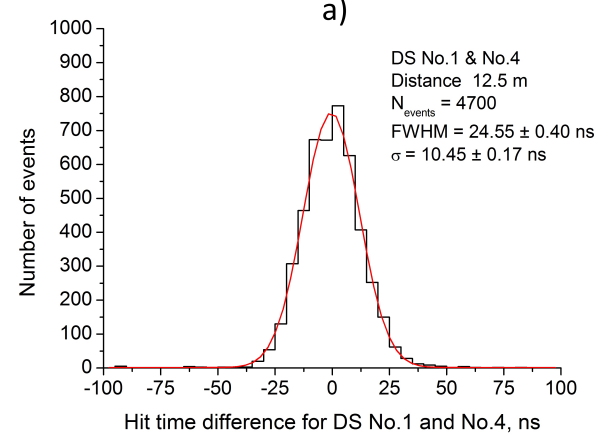

c)

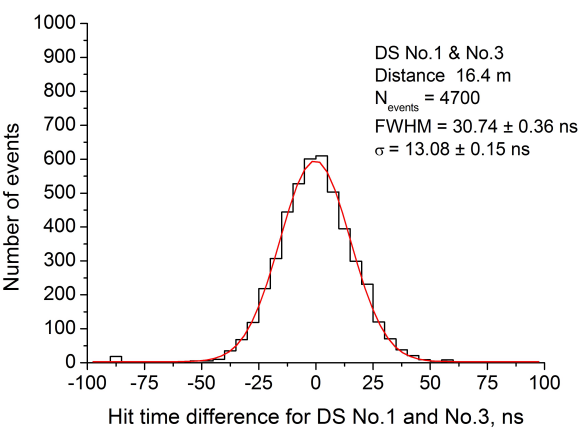

b)

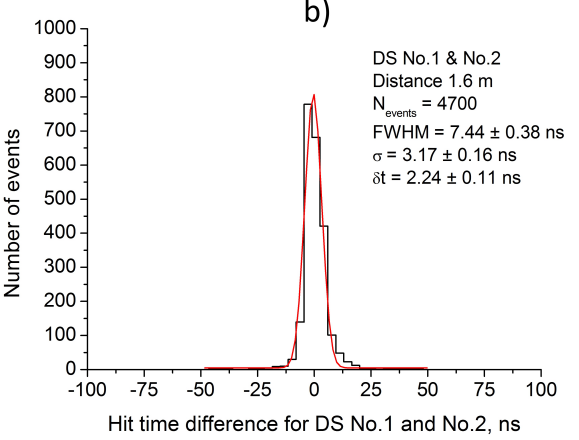

d)

Figure 8: Distribution of the hit time differences of the detector stations of the NEVOD-EAS test cluster: a) - DS No. 1 and 2, b) - DS No. 1 and 3, c) - DS No. 1 and 4, d) - DS No. 1 and 2 at a distance of $1.6 \mathrm{~m}$. 
According to the obtained data, the hit time of every detector station in a particular event was determined. Then the hit time differences between the DS pairs $(1 ; 2),(1 ; 3),(1 ; 4),(2 ; 3)$, $(2 ; 4)$ and $(3 ; 4)$ were calculated. For every pair of DS the distribution of the hit time differences was plotted (Figure $8 \mathrm{a}, \mathrm{b}, \mathrm{c}$ ). In Figure 8 only the distributions for pairs $(1 ; 2),(1 ; 3),(1 ; 4)$ are shown, as the other pairs are similar. Most of the EAS fall to the cluster from the near-vertical directions, so stations must be hit simultaneously. As seen from the Figure, the average values of the DS hit time difference distributions is close to $0 \mathrm{~ns}$. Distributions differ in width due to the different distances between the stations: $(1 ; 2)-10.6 \mathrm{~m},(1 ; 3)-16.4 \mathrm{~m},(1 ; 4)-12.5 \mathrm{~m}$.

During the estimating of the DS hit time accuracy determination, stations installed close to each other must be hit almost simultaneously. In each registered event, the hit time difference between stations 1 and 2 was determined. The obtained distribution of hit time differences for DS 1 and 2 installed close to each other is shown in Figure 8d. According to this distribution, the error of the station hit time determination is about $2.2 \mathrm{~ns}$.

\section{Conclusion}

NEVOD-EAS shower array which is being created on the basis of the Unique Scientific Facility "Experimental complex NEVOD" will allow determination of the size, the axis position and the arrival direction of the EAS. The setup will be operated in conjunction with other installations of the experimental complex NEVOD and will enable to register EAS with energies of $10^{15}-10^{17} \mathrm{eV}$, as well as to improve the technique of muon bundle energy estimation using the DECOR detector. New data obtained with NEVOD-EAS setup will allow to narrow the energy range of PCR particles responsible for generation of muon bundles with certain multiplicity arriving at different zenith angles.

\section{Acknowledgements}

The work was performed at the Unique Scientific Facility "Experimental complex NEVOD" under the Agreement on Russian-Italian cooperation with the financial support from the State provided by the Russian Ministry of Education and Science (project No. RFMEFI59114X0002).

\section{References}

[1] V.M. Aynutdinov et al., Astrophysics and Space Science, 258, 105 (1997).

[2] S.S. Khokhlov et al., Astrophys. Space Sci. Trans., 7, 271 (2011).

[3] N.S. Barbashina et al., Instrum. Exp. Tech., 43, 743 (2000).

[4] A.G. Bogdanov et al., Phys. Atom. Nucl., 73, 1862 (2010).

[5] I.A. Shulzhenko et al., J. Phys.: Conf. Ser., 409, 012098 (2013).

[6] M. Aglietta et al., Nucl. Phys. B (Proc. Suppl.), 16, 493 (1990).

[7] A. Chiavassa et al., Proc. $28^{\text {th }}$ ICRC (Tsukuba), 2, 992 (2003). 